\title{
Robust optimization of single-cycle THz setups based on phase- matching via tilted pulse fronts using an incident-fluence metric
}

\author{
Tobias Kroh ${ }^{*, a, b, c}$, Timm Rohwer ${ }^{\mathrm{a}}$, Lu Wang ${ }^{\mathrm{a}, \mathrm{b}}$, Umit Demirbas ${ }^{\mathrm{a}, \mathrm{d}}$, Huseyin Cankaya ${ }^{\mathrm{a}}$, Mikhail \\ Pergament $^{\mathrm{a}}$, Franz X. Kärtner ${ }^{\mathrm{a}, \mathrm{b}, \mathrm{c}}$ and Nicholas H. Matlis ${ }^{\mathrm{a}}$ \\ ${ }^{a}$ Center for Free Electron Laser Science (CFEL), Deutsches Elektronen-Synchrotron (DESY), \\ Notkestraße 85, 22607 Hamburg, Germany; \\ ${ }^{b}$ Universität Hamburg, Department of Physics, Luruper Chaussee 149, 22761 Hamburg, Germany; \\ ${ }^{c}$ Center for Ultrafast Imaging, Luruper Chaussee 149, 22761 Hamburg, Germany; \\ ${ }^{\mathrm{d}}$ Laser Technology Laboratory, Antalya Bilim University, 07190 Dosemealti, Antalya, Turkey
}

\begin{abstract}
Despite the popularity and ubiquitousness of the tilted-pulse-front technique for single-cycle terahertz (THz) generation, optimization of the experimental setup remains complex and difficult due to the sensitive dependence on and coupling between the optical pulse parameters, including fluence, beam size, angular dispersion and temporal compression. Here we present a systematic and robust method to tune the tilted pulse-front setup, based on use of selected multi-dimensional scans, which enables a straight-forward and accurate determination of optimum parameter values. Our methodology not only allows us to determine parameter sensitivities and achieve a robust optimum in the performance, but also enables a verification of certain physical properties of the lithium niobate prism, including the $\mathrm{THz}$ refractive index. The detailed step-by-step procedure is discussed and applied to a tilted-pulse-front $\mathrm{THz}$ setup at both room temperature and cryogenic temperatures. The procedure can be applied to any setup based on the tilted-pulse-front geometry and is important for the construction of high energy $\mathrm{THz}$ sources required for strong field terahertz applications such as novel particle acceleration schemes or beam manipulators.
\end{abstract}

Keywords: Terahertz, Nonlinear Optics, Tilted-pulse-front technique, Single-cycle THz pulses

* tobias.kroh@desy.de; Tel. +49 (0)40 8998-6359; https://ufox.cfel.de/

\section{INTRODUCTION}

Intense single-cycle terahertz $(\mathrm{THz})$ pulses have not only helped to advance fields of spectroscopy, where they can be used to probe or control many intrinsic resonances such as lattice vibrations in solids ${ }^{1,2}$, all-optical molecular alignment ${ }^{3,4}$, but have also enabled study of strong-field phenomena in the THz range unraveling both perturbative ${ }^{5-7}$ and nonperturbative extreme nonlinearities of matter ${ }^{8-10}$. Recently, the availability of strong $\mathrm{THz}$ transients has enabled the field of compact $\mathrm{THz}$ driven charged particle accelerators and manipulators to emerge which holds great promises of providing table-top accelerators and their application in science ${ }^{11}$.

Alongside flourishing fields of application, recent years have seen rapid development of table-top THz sources, including broadband $\mathrm{THz}$ pulses generated from plasmas ${ }^{12-15}$, low frequency pulses in the range of $\sim 10 \mu \mathrm{J}$ from large area semiconductor switches ${ }^{16-18}$, or spin emitter heterostructures ${ }^{19,20}$. Among the table-top sources the most common method is intra-pulse difference frequency generation (DFG) of intense ultrashort laser pulses in nonlinear materials due to both its versatility and scalability. Depending on the $\mathrm{THz}$ frequency range of interest, there is a wide range of nonlinear materials available such as semiconductors, organic crystals or ferroelectric crystals such as lithium niobate (LN) ${ }^{21}$. The latter has become a popular choice due to its availability at large $\mathrm{cm}$-sizes, high optical damage threshold, low absorption in the optical range as well as high effective nonlinear coefficient ${ }^{22}$. However, low frequency phonon contributions to the dielectric function lead to a significant difference in refractive indices in the $\mathrm{THz}$ and optical range. Thus, collinear phasematching via birefringence or temperature tuning is not possible. Consequently, methods for non-collinear phase matching were developed. Initial work in near single-cycle $\mathrm{THz}$ generation from $\mathrm{LN}$ relied on Cherenkov shockwave radiation ${ }^{23,24}$, which is problematic to scale to high energies since the pump beam needs to be focused to a narrow line below the size of 
the THz wavelength. This motivated discrete tilted intensity fronts to be used for improved THz generation first $1,2,25$. Later, Hebling and coworkers pioneered non-collinear phase-matching utilizing a pulse with a continuous intensity front tilted such that the projected group velocity of the laser pulse matched the THz wave velocity ${ }^{26}$. This allows the THz to build up coherently as it slides along the pump laser front.

In the years thereafter, the achieved $\mathrm{THz}$ pulse-energies were rapidly improved by several orders of magnitude towards the multi $\mu \mathrm{J}$-level ${ }^{22,26-29}$. First, most experiments utilized broadband Ti:Sa pump systems while later, supported by theoretical studies ${ }^{30,31}$, the focus shifted towards using more narrowband pulses with center wavelengths $>1 \mu \mathrm{m}$ associated with increased interaction lengths and reduced free-carrier generation via multi-photon absorption respectively ${ }^{32-35}$. Further improvements were enabled via cryogenic cooling of the LN crystals yielding decreased THz absorption ${ }^{36}$ and enabling the highest conversion efficiencies reported to date ${ }^{37,38}$. Others investigated the effects of chirped pump pulses ${ }^{39,40}$ or concentrated on finding optimal imaging configurations for reduced pump pulse duration variations across the tilted-pulse-front impacting the homogeneity of the generated $\mathrm{THz}$ beam ${ }^{31,41-44}$. With increasing $\mathrm{THz}$ energies generated the reported conversion efficiencies reached beyond the so called Manley-Rowe limit (THz photon number exceeds pump pulse photon number), which is possible due to cascaded DFG processes, where redshifted pump photons are effectively reused after generating a $\mathrm{THz}$ photon ${ }^{45-49}$. As phase-mismatch is less pronounced for redshifted pump photons, the DFG process is dominant compared to the opposite process where repeated sum-frequency-generation of pump-THz photons would annihilate $\mathrm{THz}$ photons instead, thus leading to overall enhanced $\mathrm{THz}$ conversion. The broadening of the pump pulse via this cascaded nonlinear interaction in combination with the group-velocity dispersion (GVD) associated with angular dispersion necessary to tilt the pulse imposes the main limitation for the maximum efficiency of the process ${ }^{50}$. Via development of increasingly advanced simulation tools to study the complex spatiotemporal effects in tilted-pulse-front setups ${ }^{22,51-53}$, further limiting effects such as THz absorption by free carriers ${ }^{54}$, selfphase modulation leading to phase-mismatch ${ }^{34}$ or stimulated Raman scattering enhancing the breakup of the pump pulse 55 were identified. In order to circumvent some of these limitations, several modified setup schemes were investigated, promising to allow scaling to even higher $\mathrm{THz}$ energies ${ }^{43,56-58}$. With reported pulse energies up to $>400 \mu \mathrm{J}{ }^{38}$ the conventional tilted-pulse front setup remains unbeaten and promises to enable single-cycle $\mathrm{THz}$ pulses with energies of mJ-level.

Despite the popularity and ubiquitousness of the tilted-pulse-front technique, optimization of the setup remains complex and difficult due to the sensitive dependence on and coupling between the optical pulse parameters, including fluence, beam size, angular dispersion and temporal compression of the pump pulse across the tilted intensity front. To the best of our knowledge, the literature lacks systematic optimization studies focused on tilted pulse front setups. In addition, there is a lack of experimental characterizations of important parameters such as imaging magnification and optimized pulsefront-tilt. Thus, it remains challenging to compare different schemes reported in the literature. In this work, the setup was designed and built to decouple most of the important parameters and determine an optimum configuration for a given laser pulse duration and energy via selected multi-dimensional parameter scans. In addition, characterization techniques in order to improve the comparability of different tilted-pulse-front setups are proposed and conducted. The obtained data allows to determine sensitivities of parameters on both the efficiency and the THz beam profile. Based on the findings, a step-bystep procedure of how to perform the setup optimization is given.

The manuscript is organized as follows. In the first section, the design strategy and the experimental setup are introduced. In the second part, the individual parameter scans and measurements are motivated alongside the discussion of the stepby-step optimization procedure proposed. The section concludes with a discussion of the results and parameter sensitivities.

\section{EXPERIMENTAL SETUP}

In order to allow a systematic optimization of a setup relying on a tilted-pulse-front based non-collinear phase-matching, the first step is to ensure the setup design allows for such optimization to be carried out and that the tuning parameters are decoupled to a reasonable extend. First, the optical design is discussed, before describing the implemented setup as well as important characterization techniques that will improve comparability with other setups. Moreover, a set of selected multi-dimensional parameter scans for optimizing the setup is motivated and discussed. 


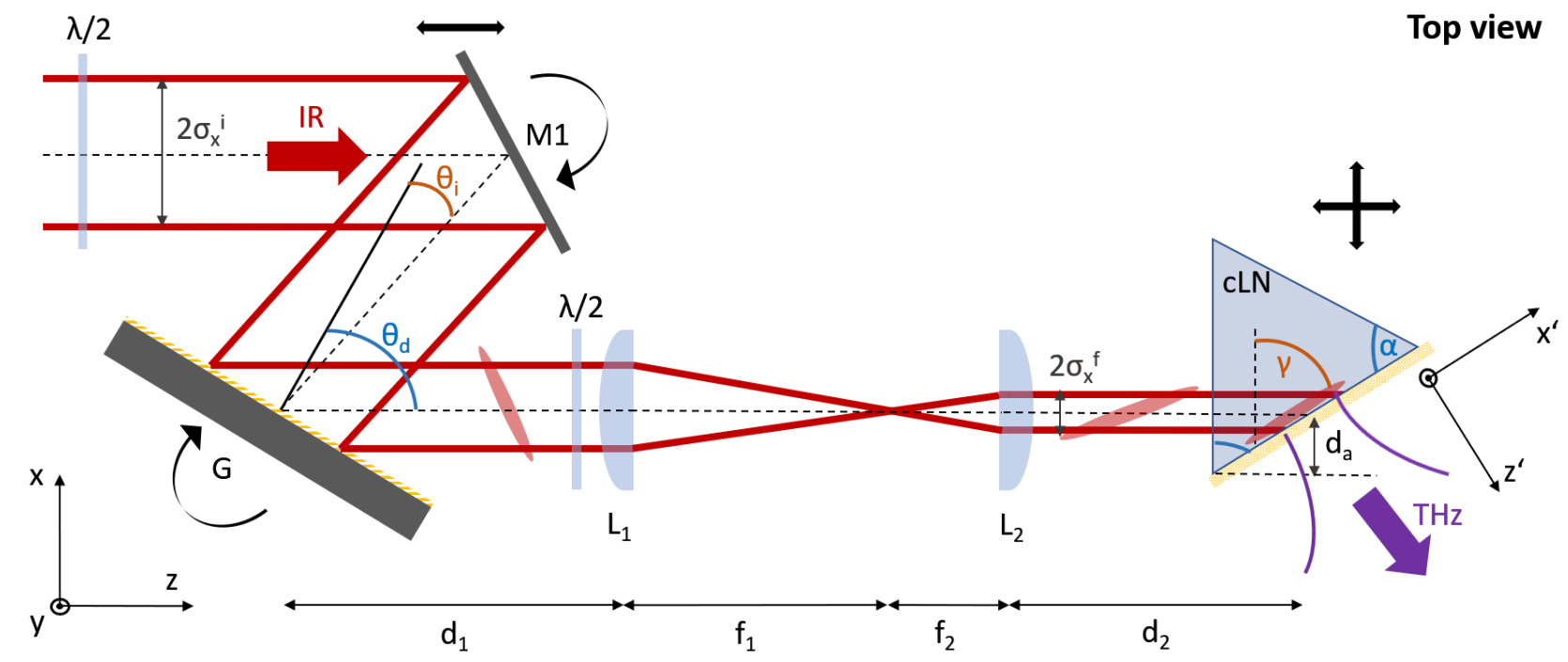

Figure 1. Schematic of the experimental setup. The pulse front is tilted via a gold-coated holographic diffraction grating $(\mathrm{G})$, while a cylindrical telescope $\left(\mathrm{L}_{1}, \mathrm{~L}_{2}\right)$ was employed to recompress the pulse inside the $5 \% \mathrm{MgO}$ doped congruent lithium niobate (cLN) crystal. Two half-wave-plates allowed to adjust the pump laser polarization such that both diffraction efficiency of the grating and effective nonlinear coefficient of the intra-pulse DFG process were maximized. In order to optimize the performance of the setup, the pump beam waist $\sigma_{\mathrm{x}}{ }^{\mathrm{i}}$, the grating angle $\left(\theta_{\mathrm{i}}, \theta_{\mathrm{d}}\right)$ and thus the pulse front tilt angle $\gamma$, the pump energy $\mathrm{E}_{\mathrm{p}}$ as well as the crystal position along $\mathrm{x}$ and $\mathrm{z}$-axis were systematically scanned.

\subsection{Setup design: A practical view on choosing a proper imaging setup for the optimization procedure}

Two important parameters for the design of a tilted pulse front setup are the tilt-angle $\gamma$ of the intensity front (see Fig. 1) as well as the angle of the grating image inside the crystal $\gamma_{\mathrm{im}}$. While the correct pulse front tilt ensures noncollinear phasematching of the intra-pulse DFG process, the image location of the grating defines the region across which the pump pulse duration is re-constructed after its spatio-temporal broadening due to the angular dispersion imposed on the beam by the grating. Both angles sensitively depend on the choice of grating parameters such as the groove density $\rho$, angle of diffraction $\theta_{\mathrm{d}}$ as well as the magnification factor $M$ of the imaging setup ${ }^{31,41,42,59}$ :

$$
\begin{aligned}
\tan \gamma & =\frac{m \lambda_{0} \rho}{M n_{g} \cos \theta_{d}} \\
\tan \gamma_{i m} & =M n_{g} \tan \theta_{d}
\end{aligned}
$$

Here, $m$ denotes the order of diffraction picked from the grating (usually $m= \pm 1$ ), $\lambda_{0}$ the center wavelength of pump laser and $n_{\mathrm{g}}$ the optical group index of LN. In order to optimize the symmetry and quality of the generated THz pulses, $\gamma$ should equal $\gamma_{\mathrm{im}}$. As the pulse-front tilt has to be chosen according to $\cos \gamma \approx \mathrm{n}_{\mathrm{g}} / \mathrm{n}_{\mathrm{THz}}$ in order to fulfill the non-collinear phasematching condition ${ }^{26}$, Eqs. (1) and (2) recommend selected pairs of $\rho$ and $\mathrm{M}$ for optical setups.

Among the frequently used configurations for tilted-pulse front setups for $\mathrm{THz}$ generation, there are two versions of imaging setups, which are widely used. Most setups utilize a single lens for imaging the tilted-pulse front into the crystal whereas others rely on a telescope for imaging. One advantage of the single-lens setup is, that the magnification and thus both the pulse-front-tilt angle and the angle of the image can be tuned via adjusting the grating-lens and lens-crystal distances, often used as optimization procedure in experiments $34,35,38,58,60$. However, it is harder to decouple effects due to a change in pump fluence from effects caused by the altered angles of pulse-front tilt and image plane during this optimization procedure and determine a global optimum of the THz efficiency since all three parameters are simultaneously changed. Imaging telescopes yield the advantage of a fixed magnification factor $M$ independent of the distance of the position of the object and that they can be characterized with better precision. Thus, once constructed and characterized, it is easier to keep track of the fluence on the crystal and this way decouple effects caused by the pulse-front tilt from a 
change in pump fluence. Additionally, single-lens setups have been reported to suffer more severely from imaging errors degrading the pump pulse duration across the intensity front and thus degrading the homogeneity and quality of the generated $\mathrm{THz}$ beam ${ }^{43,44}$. Thus, in order to achieve reduced imaging errors, a more accurately characterized setup as well as easier decoupling of scanning parameters, a two-lens telescope was chosen for the setup shown in Fig. 1. Note that in such a setup, the pulse-front tilt angle required for phase-matching is then optimized via the grating angle, which has the slight disadvantage that it can be more tedious to scan.

As the non-collinear phase-matching takes place in the $\mathrm{x}-\mathrm{z}$ plane, there is an optimal pump beam size $\sigma_{\mathrm{x}}{ }^{\mathrm{f}}$ (see Fig. 1) associated with an optimum interaction length between pump and $\mathrm{THz}$ beam. The beam width along the $\mathrm{y}$-axis however is not directly affecting the phase-matching process and thus allows adjustment of the pump fluence to its optimal level. Additionally, given a large crystal, this beam size $\sigma_{\mathrm{x}}{ }^{\mathrm{f}}$ can be increased for scaling tilted-pulse-front setups to higher pump energies. Thus, cylindrical optics for individual adjustment of both $\sigma_{\mathrm{x}}{ }^{\mathrm{f}}$ and $\sigma_{\mathrm{y}}{ }^{\mathrm{f}}$ were chosen for the optimization procedure in this work.

The imaging setup and diffraction grating for the setup were selected following the suggestions given in Refs. ${ }^{31,41,42,59}$. A holographic diffraction grating was implemented as it yields the advantage of an almost flat diffraction efficiency across a wide range of angles. This is particularly helpful for scans of the grating angle in order to tune the pulse front tilt without affecting the pump energy incident onto the crystal significantly. To approximately achieve a well compressed pulse duration across the tilted intensity front inside the crystal, combinations of $M=f_{2} / f_{1}$ (note, in some literature the angular magnification $1 / \mathrm{M}$ is used instead) and grating period $\rho$ should be chosen, such that $\gamma=\gamma_{\mathrm{im}}$. Table 1 summarizes the design chosen for the setup presented in this work.

Table 1. Parameters used for the design and the ones found for the experimentally optimized tilted-pulse-front setup used in this work for both cryogenic temperatures $(80 \mathrm{~K})$ as well as room temperature (RT).

\begin{tabular}{|l|l|l|}
\hline \multicolumn{1}{|c|}{ Parameter } & \multicolumn{1}{c|}{ Design } & \multicolumn{1}{c|}{ Experimental results } \\
\hline Groove density $\rho$ & 1500 lines/mm & 1500 lines/mm \\
\hline Center wavelength $\lambda_{0}$ & $1020 \mathrm{~nm}$ & $1020 \mathrm{~nm}$ \\
\hline Telescope magnification $\mathrm{M}$ & 0.6666 & $0.6721 \pm 0.0007$ \\
\hline Incident angle $\theta_{\mathrm{i}}$ & $42.8^{\circ}(80 \mathrm{~K}), 41.0^{\circ}(\mathrm{RT})$ & $41.52^{\circ}(80 \mathrm{~K}), 39.96^{\circ}(\mathrm{RT})$ \\
\hline Diffraction angle $\theta_{\mathrm{d}}$ & $58.2^{\circ}(80 \mathrm{~K}), 60.9^{\circ}(\mathrm{RT})$ & $59.7^{\circ}(80 \mathrm{~K}), 62.3^{\circ}(\mathrm{RT})$ \\
\hline $\mathrm{n}_{\mathrm{gr}}(1020 \mathrm{~nm})$ & $2.2158^{*}(80 \mathrm{~K}), 2.2316(\mathrm{RT}){ }^{61}$ & \\
\hline $\mathrm{n}_{\mathrm{THz}}$ & $4.89^{* *}(80 \mathrm{~K}), 5.21^{* *}(\mathrm{RT})$ & $0.67(80 \mathrm{~K}), 0.61(\mathrm{RT})$ \\
\hline Grating magnification $\mathrm{M}_{\mathrm{gr}}$ & $0.48(80 \mathrm{~K}),(\mathrm{RT})$ & $63.7^{\circ}(80 \mathrm{~K}), 65.4^{\circ}(\mathrm{RT})$ \\
\hline Pulse-front-tilt angle $\gamma$ & $63.0^{\circ}(80 \mathrm{~K}), 64.8^{\circ}(\mathrm{RT})$ & $62.4^{\circ}$ \\
\hline Crystal cut angle $\alpha$ & $63.0^{\circ}(80 \mathrm{~K}), 64.8^{\circ}(\mathrm{RT})$ & \multicolumn{2}{|c|}{} \\
\hline
\end{tabular}

* obtained via extrapolation of measured values given in ${ }^{62}$

** obtained from $\mathrm{THz}$ time-domain spectroscopy measurements at $\sim 300 \mathrm{GHz}$

*** Estimated based on inserting the obtained experimental results into Eq. (1).

\subsection{Experimental setup \& measurement devices}

The setup geometry implemented is shown in Fig. 1. A 2-stage cryogenic Yb:YLF amplifier system delivering high energy pulses centered at $\sim 1020 \mathrm{~nm}$ with a pulse duration of $\sim 1.35 \mathrm{ps}$ at $10 \mathrm{~Hz}$ repetition rate was used as pump source ${ }^{63}$. A multi$\mathrm{kHz}$ repetition rate version of this amplifier, potentially with sub-250-fs pulse duration is under development for future experiments ${ }^{64}$. For the experiments, up to around $\sim 25 \mathrm{~mJ}$ were utilized and sent onto a gold-coated holographic diffraction grating (Spectrogon AB, 1500 1/mm, 50x50 mm, > $90 \%$ efficiency for $1020-1032 \mathrm{~nm}$, deviation $13^{\circ}$ ) mounted on a hybrid rotation stage with integrated translation capability $\left(\right.$ Newport, DGM-1). The diffracted beam of $-1^{\text {st }}$ order $(\mathrm{m}=-1)$ was sent through a Keplerian cylindrical telescope $\left(\mathrm{f}_{1}=300 \mathrm{~mm}, \mathrm{f}_{2}=200 \mathrm{~mm}\right.$, Thorlabs, 1" diameter, B-coated) that images the grating into the $5 \% \mathrm{MgO}$ doped congruent lithium niobate $(\mathrm{LN})$ crystal $(27 \times 27 \times 25 \mathrm{~mm}$, height $25 \mathrm{~mm}$, cut 
angle $\alpha \sim 62.4^{\circ}$ ), if the distance $\mathrm{d}_{2}$ (second lens-crystal) is set correctly depending on $\mathrm{d}_{1}$ (grating-first lens). The LN crystal was AR-coated on the two surfaces where the pump beam enters and exits the crystal, while the THz output surface remained uncoated. The positioning of the crystal within the pump beam was performed using a motorized stage (Thorlabs, LTS150) for movement along the $\mathrm{z}$-axis and a manual translation stage for positioning along the $\mathrm{x}$-axis. Two half-waveplates before and after the grating ensured optimal polarization for highest diffraction efficiency (TM polarization) and maximized effective nonlinear coefficient $d_{\text {eff }}$ of the intra-pulse-DFG process, which is approximately proportional to the $4^{\text {th }}$ power of the electric field projected onto the optical axis of the LN crystal and thus shows a cos ${ }^{4}$-dependence on the polarization angle (see Fig. 3b).

A motorized $\lambda / 2$ waveplate (Thorlabs, PRM1Z8) in combination with a thin-film-polarizer (TFP) were implemented to automate pump energy scans. The incident IR pump energy was measured using a large area energy meter (Coherent Inc., LabMax TOP with sensor EnergyMax-USB J-50MB-LE) while the extracted THz pulse energies were measured using two different pyro-electric detectors: A sensitive detector with a $9 \mathrm{~mm}$ active area (Gentec-EO, SDX-1152 with MT coating) without traceable calibration in the frequency range $<4 \mathrm{THz}$, and a less sensitive but larger area detector (SLT Sensor- und Lasertechnik GmbH, THz 20, $20 \mathrm{~mm}$ active area) with flat frequency response across the whole THz range, crucial for characterizing and optimizing broadband THz-pulses, with traceable calibration at $693 \mathrm{GHz}$ performed at the PTB Berlin, Germany (calibration certificate 73426 PTB 18). A cross-calibration of both detectors with broadband THz pulses centered around $200 \mathrm{GHz}$ yielded a calibration factor of $(74 \pm 3) \mathrm{mV} / \mu \mathrm{J}$ for the Gentec detector, from which the signal was acquired using a digital storage oscilloscope (Tektronix, TDS 2024C). In both cases, the THz energy was acquired automatically via a desktop computer and home-built software. To avoid distortions of the measurement, residual infrared and second harmonic light exiting the LN was blocked upstream of the detectors by a sheet of black polyethylene (PE, 1.9mm thickness) with a measured average transmission of $\sim 50 \%$ in the $150-450 \mathrm{GHz}$ range by THz time-domain spectroscopy, which agrees well with the transmission of $\sim 54 \%$ determined throughout the detector cross-calibration. The transmission factor of 54\% was used in order to obtain the THz energy in front of the PE covering the detector.

Experiments were conducted both at room- and cryogenic temperatures, for each of which there were slight modifications: At room temperature, a three-layered Kapton-tape (Thorlabs, KAP22-075) coating was applied to the THz output surface of the crystal in order to reduce transmission losses. In addition, the crystal was indium bonded to a copper cold-finger inside a vacuum chamber attached to a liquid nitrogen Dewar in order to be cooled to $\sim 80 \mathrm{~K}$. At cryogenic temperature, no AR-coating for $\mathrm{THz}$ was applied and the usable $\mathrm{THz}$ energy was measured outside the vacuum chamber after transmission through a $3 \mathrm{~mm}$ thick uncoated TPX window (TYDEX).

As there is still a lack of reliable $\mathrm{THz}$ cameras for the characterization of broadband pulses in the spectral range $<1 \mathrm{THz}$ with sufficient resolution, a motorized x'-y'-z' scanner (x', y': Thorlabs, NRT 100-M, z': Thorlabs, LTS300) was constructed in order to scan a sensitive small-area pyroelectric detector (WiredSense GmbH, MPY-01-XX and MPY-LG$\mathrm{XX}, 2 \times 2 \mathrm{~mm}$ active area) across the $\mathrm{THz}$ beam profile and reconstruct images of the $\mathrm{THz}$ beam. The detector signal was acquired using a fast-readout digital oscilloscope (Pico Technology, PicoScope 2408B) via home-built software, with integration and background subtraction implemented for reduced SNR. The same sensitive detector was used in a Michelson type $\mathrm{THz}$ interferometer utilizing a pellicle beam splitter in order to measure the THz spectrum via Fourier transformation of the recorded interferogram.

As mentioned in section 2.1, the pump width $\sigma_{\mathrm{x}}{ }^{\mathrm{i}}$ in the phase-matching plane (x-z-plane) was adjusted via different Galilean cylindrical telescopes that were set up before the tilted-pulse-front setup. The pump beam sizes along $\mathrm{x}\left(\sigma_{\mathrm{x}}{ }^{\mathrm{i}}\right)$ and $\mathrm{y}$-axis $\left(\sigma_{y}{ }^{i}\right)$ were monitored using a large area CCD camera (DataRay Inc., WinCamD-LCM-C), while the telescope characterization described in the next section was performed using a smaller CCD camera (Ophir, Spiricon USB-SP 926) and a CW alignment laser (CrystalLaser Inc., $1030 \mathrm{~nm}$ wavelength).

\section{STEP-BY-STEP OPTIMIZATION PROCEDURE}

Given fixed laser parameters, the nonlinear process underlying the $\mathrm{THz}$ generation depends heavily on phase-matching required for efficient buildup of the $\mathrm{THz}$ and on the effective interaction length between pump pulse and $\mathrm{THz}$ pulse. In addition, the temporal compression of the pump pulse in the interaction volume and the peak fluence impact the THz generation. Consequently, optimization involves tuning the pulse-front tilt angle $\gamma$ (phase-matching), the transverse size of the beam in the pulse-front-tilt plane $\sigma_{\mathrm{x}}{ }_{\mathrm{f}}$ alongside with setting the transverse position, $\mathrm{d}_{\mathrm{a}}$, of the beam relative to the crystal apex (which determines the effective interaction length), setting the longitudinal position of the crystal relative to the image 
plane (temporal compression) and finally adjusting the transverse size of the beam in the vertical, non-tilt plane $\sigma_{\mathrm{y}}{ }^{\mathrm{f}}$ (optimal fluence). Fig 2(a) provides an overview over these steps needed for the optimization.

a)

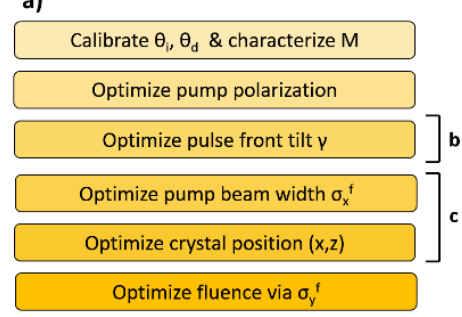

b)

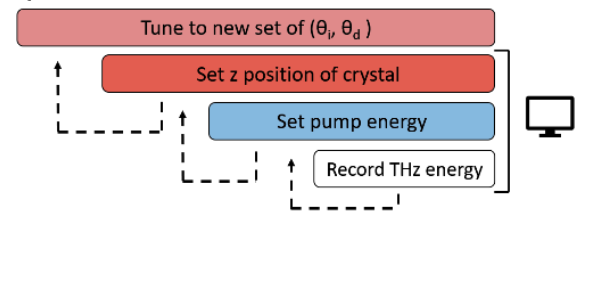

c)

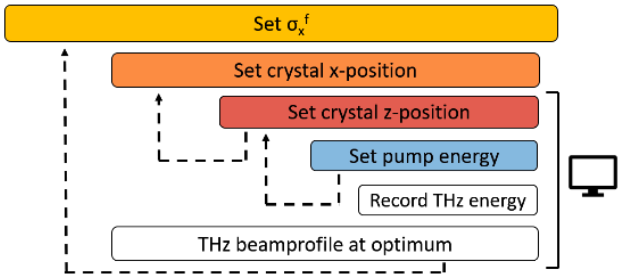

Figure 2. (a) Schematic of the experimental optimization procedure performed with in-depth schematics showing the multiparameter scans necessary to optimize the pulse-front tilt angle $\gamma(\mathrm{b})$ and the transverse pump beam size $\sigma_{\mathrm{x}}{ }^{\mathrm{f}}$ and the crystal position (c). Frequently needed scans, where automation is useful, are visualized via the computer icon.

Some steps are rather simple, such as maximizing the effective nonlinear coefficient for the DFG process via alignment of the pump polarization along the optical crystal axis, as shown in Fig. 3(b). Nonetheless, this step is important as the THz energy is sensitively dependent on the pump polarization and only a few degrees of mistuning lead to significant degradation of the efficiency.

\subsection{Optimization of the pulse front tilt}

While some steps are simple, other steps, however, require up to several parameters to be tuned at once, as some affect one another. For example, adjusting the pulse-front can be performed via turning the diffraction grating. In this process however, both diffraction efficiency and magnification factor of the grating change, thus affecting the pump fluence incident on the crystal. Therefore, the pump energy must be adjusted to keep the pump fluence at a constant level in order to compare results obtained from different grating angles. Alternatively, it is possible to scan the energy for each new grating angle and compare the measurements at the same fluence level later, which is the procedure chosen here (see. Fig. 2(b)). As the image location of the grating may be slightly affected during the scan, the crystal-lens distance $\mathrm{d}_{2}$ was additionally scanned, which can be considered optional in the overall optimization strategy, though. Note, that optimizing the pulse front tilt ideally requires a sufficiently large pump size $\sigma_{\mathrm{x}}{ }^{\mathrm{f}}$ on the order of the effective interaction length $(>1 \mathrm{~mm})$, as for small beam sizes phase-matching matters less and the dependence on the pulse-front tilt is less pronounced ${ }^{53,65}$. On the other hand, the absolute changes in the beam size via the varying grating magnification $\mathbf{M}_{\mathrm{gr}}$ can distort the optimization, which becomes more severe for larger beams. As such, large changes in $\sigma_{\mathrm{x}}{ }^{\mathrm{f}}$ could distort the optimization of gamma via the affected interaction length and in addition, the apex-distance $\mathrm{d}_{\mathrm{a}}$ would have to be readjusted for each new grating angle, increasing the complexity of the procedure.

In preparation for the grating angle optimization, it is crucial to calibrate the grating mount angle to the actual set of $\left(\theta_{\mathrm{i}}, \theta_{\mathrm{d}}\right)$ and to characterize the imaging setup in place. Firstly, this allows comfortable pump fluence determination at the crystal position from measured beam profiles before the setup and secondly, to determine the pulse-front tilt found optimal (or angular dispersion respectively). The latter can be used to compare one tilted-pulse-front setup with another. The rotation angle $\theta_{\text {mount }}$ can be calibrated via the angle at which the zero-order beam is diffracted back onto itself $\left(\theta_{\text {mount }}{ }^{0}\right)$. The calibrated angle of incidence is obtained via $\theta_{\mathrm{i}}{ }^{\mathrm{cal}}=\theta_{\text {mount }}-\theta_{\text {mount }}{ }^{0}$, while $\theta_{\mathrm{d}}$ is obtained via the grating equation. If the grating is now rotated by an angle $\Delta \theta_{\text {mount, then }} \theta_{\mathrm{d}}$ is rotated by the same amount, while this time $\theta_{\mathrm{i}}$ is obtained via the grating equation. Note, that since $\theta_{\mathrm{d}} \neq \theta_{\mathrm{i}}$, the grating (de)magnifies the beam along the $\mathrm{x}$-axis via $\mathbf{M}_{\mathrm{gr}}=\cos \theta_{\mathrm{d}} / \cos \theta_{\mathrm{i}}$.

The telescope on the other hand can be effectively characterized by its magnification $\mathrm{M}$, on which both the pulse front tilt and image-plane angle depend (see Eqs. (1) and (2)). Therefore, it is crucial to measure M with high precision. A simple yet accurate measurement technique is to image an object placed on a micrometer stage at distance $\mathrm{d}_{1}$ before the telescope onto a CCD camera mounted on a stage at distance $\mathrm{d}_{2}$ after the second lens. Using the ray-tracing matrix formalism it can easily be shown that $\Delta \mathrm{d}_{2}=\mathrm{M}^{2} \Delta \mathrm{d}_{1}$. Thus, recording the positions $\mathrm{d}_{2}$ where the image is sharp vs various positions $\mathrm{d}_{1}$ of the 
object, allows measurement of $\mathrm{M}^{2}$ via the slope of a linear fit to the data with orders of magnitude higher precision as compared to using a simple resolution test target. For the setup discussed here, $\mathbf{M}=0.6721 \pm 0.0007$ as shown in Fig. 3(a).

a)

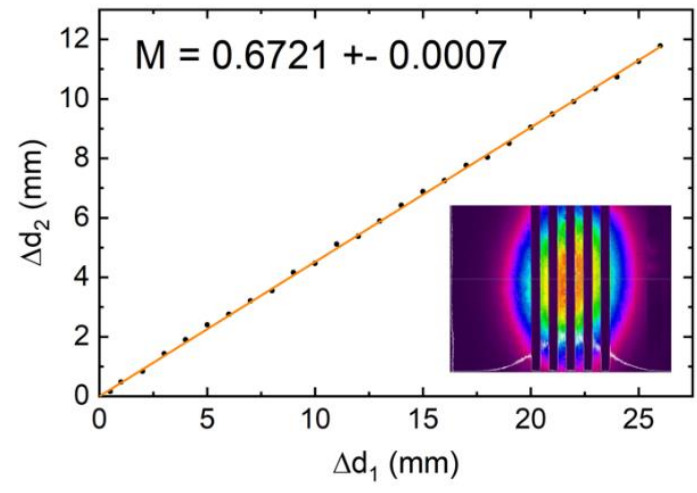

b)

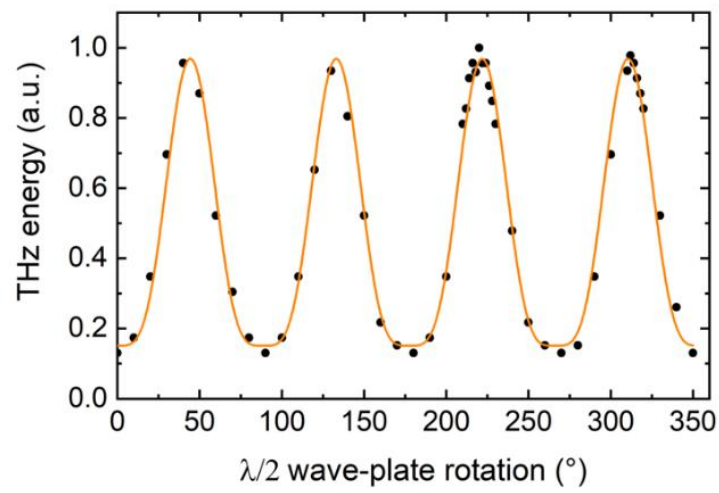

Figure 3. (a) Determination of the imaging setup magnification $M$ via recording the shift $\Delta \mathrm{d}_{2}$ of the image position of a resolution test target (see insert) for known longitudinal displacements of the object before the telescope $\Delta \mathrm{d}_{2}$. The magnification factor $M$ can then be determined via a linear fit (orange) to the data. (b) Shows the optimization procedure of the pump polarization via the angle of the half-wave-plate after the grating. In agreement with expectations the recorded $\mathrm{THz}$ energy follows a $\sim \cos ^{4}$ law with the angle of the pump electric field polarization relative to the optical axis of the crystal. Fitting to the data allows effective and precise determination of the optimum waveplate angle.

With $\mathrm{M}$ and $\mathrm{M}_{\mathrm{gr}}$ known, the pump peak fluence $\mathrm{F}_{\mathrm{p}}$ incident on the crystal can then be obtained using the characteristics of the pulse before the grating:

$$
F_{p}=\frac{T_{\text {setup }} E_{p}^{i}}{\pi M_{g r} M M_{x} \sigma_{x}^{i} M_{y} \sigma_{y}^{i}}
$$

Where $E_{p}{ }^{i}$ is the incident pulse energy onto the grating, $\sigma_{x}{ }^{i}$ and $\sigma_{y}{ }^{i}$ denote the $1 / e$ radii of the intensity profile, $T_{\text {setup }}$ is the combined transmission through the setup including transmission through the telescope as well as the diffraction efficiency of the grating. $\mathrm{T}_{\text {setup }}$ was measured to be surprisingly high for the presented setup with around $96 \%$ and constant over the range of angles scanned. $\mathrm{M}_{\mathrm{x}}$ and $\mathrm{M}_{\mathrm{y}}$ are the magnification factors of cylindrical telescopes used to shape the beam before the tilted-pulse-front setup. So far $\mathrm{M}_{\mathrm{x}}=\mathrm{M}_{\mathrm{y}}=1$. Fig 4 shows the sensitive dependence of the measured THz energy on both pump fluence and $\theta_{\mathrm{i}}$ for operation at both room and cryogenic temperature $(80 \mathrm{~K})$. The optimal angle differs, since the refractive index in the THz range changes as the crystal is cooled to lower temperatures, thus requiring a change in pulsefront tilt for phase-matching. Note, that even though at cryogenic temperatures the pump fluence was kept low to avoid optical damage during the scans, the achieved energies are comparable to the room-temperature setup pumped at almost twice the fluence. Both scans show how even a small deviation from the optimal pulse-front tilt affects the THz conversion efficiency. The optima found also allow for a rough estimate of the THz refractive indices. Using Eqs. (1) and (2), assuming an optimal setup configuration and inserting all known parameters except the $\mathrm{THz}$ indices, it is possible to obtain an estimate for their values in the setup (see Tab. 1). The estimated refractive indices are within a range of approximately \pm 0.2 compared to published values ${ }^{66,67}$. 
a)

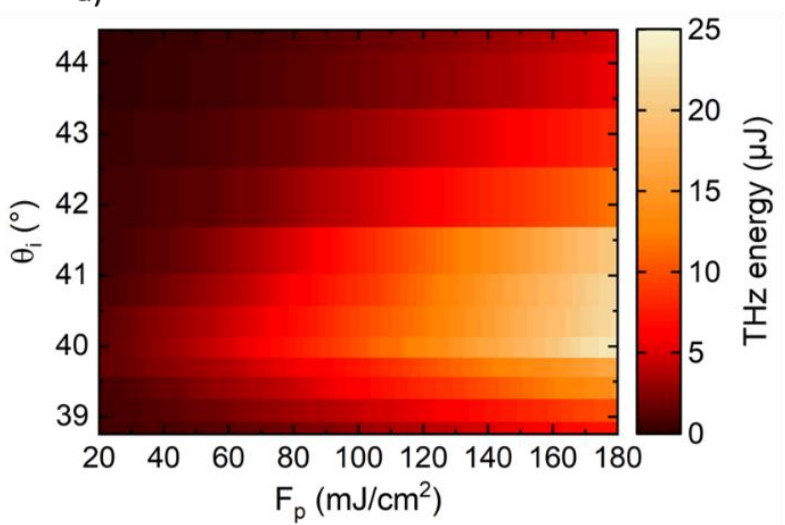

b)

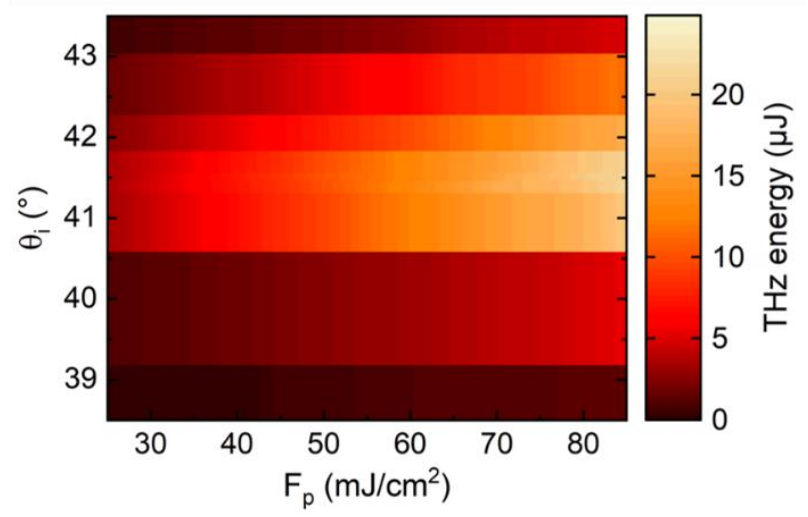

Figure 4. Optimization of the pulse front tilt angle via tuning of the grating angle at room-temperature (a) and with a cryogenically cooled LN crystal (b). The THz energies plotted were measured outside the crystal or Dewar respectively.

\subsection{Optimizing the interaction volume of the nonlinear process}

After the grating angle is set to the optimum found, the interaction length is to be optimized next. As can be seen from Fig. $2(\mathrm{c})$, optimizing $\sigma_{\mathrm{x}}{ }^{\mathrm{f}}$ requires the crystal position within the pump beam to be optimized each time. Energy scans are required in addition, as the fluence would otherwise be different for each $\sigma_{\mathrm{x}}{ }^{\mathrm{f}}$. In order to test different pump sizes, 5 different cylindrical telescopes with different $\mathrm{M}_{\mathrm{x}}$ were constructed in front of the tilted-pulse-front setup. For scans of the crystal position it proved helpful to use a large area $\mathrm{THz}$ detector, as otherwise the detector position had to be constantly adjusted due to the THz output location changing with crystal position. For each $\sigma_{\mathrm{x}}{ }^{\mathrm{f}}\left(1 / \mathrm{e}^{2}\right.$ intensity radius) the maximal observed $\mathrm{THz}$ energy at $\mathrm{F}_{\mathrm{p}}=90 \mathrm{~mJ} / \mathrm{cm}^{2}$ is shown in Fig. 5(a) for the setup operated at room temperature. The corresponding optimum distance $d_{a}$ at each tested $\sigma_{x}{ }^{f}$ was found to lie within the range of $3-4 \mathrm{~mm}$, while the accuracy of the measurement was $\sim 0.5 \mathrm{~mm}$. The global optimum is observed at $\sigma_{\mathrm{x}}{ }^{\mathrm{f}} \approx 2.5 \mathrm{~mm}\left(1 / \mathrm{e}^{2}\right.$ intensity radius) and $\mathrm{d}_{\mathrm{a}}=4 \pm 0.5 \mathrm{~mm}$. Fig 5 (b) shows the recorded $\mathrm{THz}$ energy and conversion efficiency as a function of the pump fluence for the setup operated at room temperature. $\mathrm{THz}$ pulses with usable energies up to $\sim 30 \mu \mathrm{J}$ at a conversion efficiency $\sim 0.12 \%$ were generated while pumping with a fluence up to $160 \mathrm{~mJ} / \mathrm{cm}^{2}$.

The impact of the crystal position onto the THz energy is shown in Fig. $5(\mathrm{c})$ for $\sigma_{\mathrm{x}}{ }^{\mathrm{f}} \approx 1.4 \mathrm{~mm}$. Though $\mathrm{d}_{\mathrm{a}}$ and $\mathrm{d}_{2}$ are shown not to be independent of each other, in principle they can be optimized individually because the range within which the THz energy would degrade significantly is on the order of one mm. Thus, a detailed 2D scan of $d_{a}$ vs $d_{2}$, as shown in Fig. $5(c)$, is not necessary for a rough optimization. As can be seen from Fig. 5(d), the distance $d_{a}$ affects the THz energy more severely at smaller values. If the beam is set completely onto the crystal, the impact onto the THz energy is less pronounced. We note that recent numerical studies have revealed the temporal $\mathrm{THz}$ waveform to degrade from a near single-cycle pulse for pump beams offset far from the crystal apex, even if the energy may not be affected much ${ }^{68}$. As such it is recommended to stay as close to the crystal apex as possible while maintaining reasonable efficiency levels.

Note, that in addition it is important to perform these scans at reasonable fluence levels, since optimization at low fluences may yield different optimal configurations in comparison to high fluences due to the different effective interaction lengths ${ }^{53}$. The trend seen in Fig 5(a) verifies the existence of an optimal pump beam width so far only predicted by simulations. Small beams do not suffer as much from imperfect imaging conditions, but the THz may not build up effectively if the interaction length is larger. Larger pump beams however suffer from more severe temporal distortions due to imaging errors and parts of the beam going through more material degrade due to breakup of the pulse, thus not contributing to the $\mathrm{THz}$ generation effectively anymore ${ }^{53,69}$. 
a)

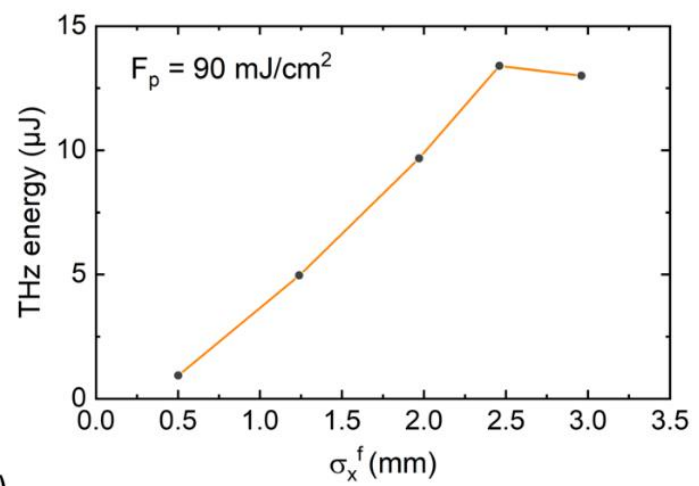

c)

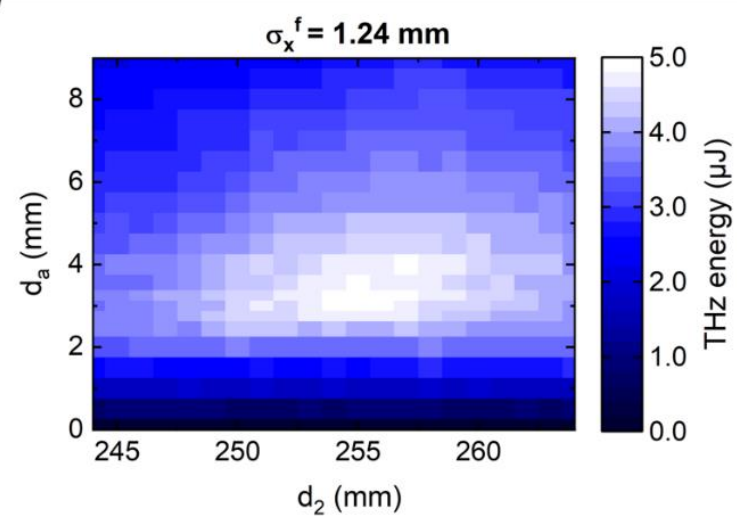

b)

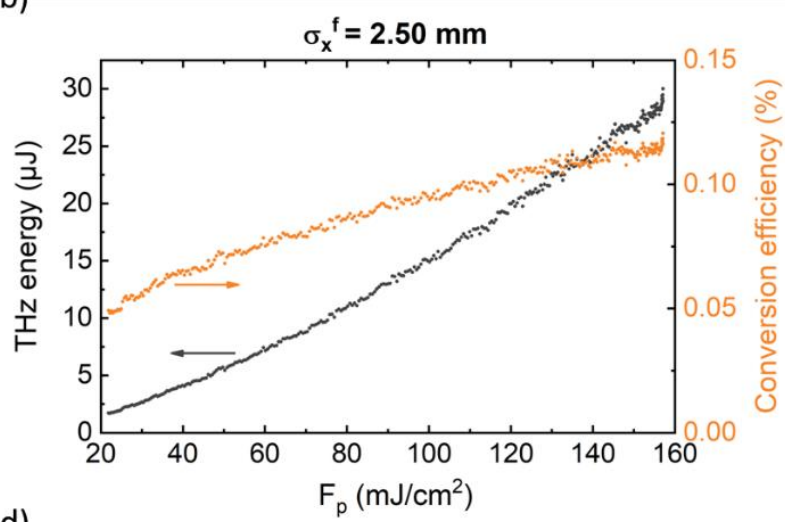

d)

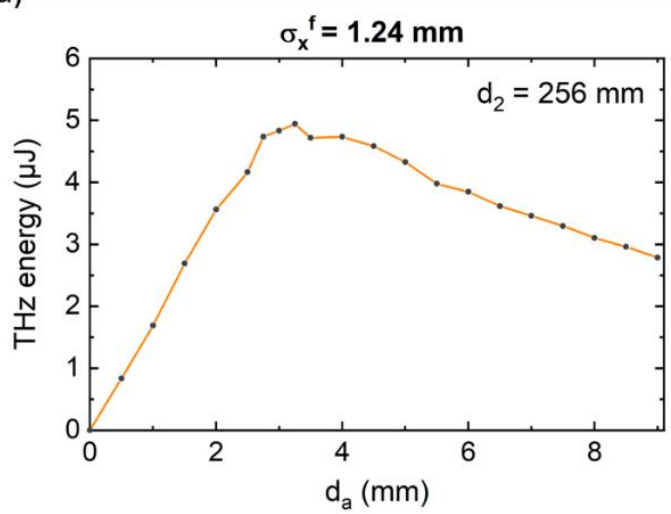

Figure 5. (a) The maximum THz energy (determined at room temperature) strongly increases with pump beam radius $\sigma_{\mathrm{x}}{ }^{\mathrm{f}}$ and saturates for $\sigma_{\mathrm{x}}{ }_{\mathrm{f}} \approx 2.5 \mathrm{~mm}\left(1 / \mathrm{e}^{2}\right.$ intensity). The measurements were compared for constant fluence of $\mathrm{F}_{\mathrm{p}}=90 \mathrm{~mJ} / \mathrm{cm}^{2}$. (b) THz pulses with energies up to $\sim 30 \mu \mathrm{J}$ at a conversion efficiency $\sim 0.12 \%$ were generated pumping with $\sigma_{\mathrm{x}}{ }^{\mathrm{f}}=2.5 \mathrm{~mm}$ and a fluence up to $160 \mathrm{~mJ} / \mathrm{cm}^{2}$. (c) The THz energy (here $F_{p}=90 \mathrm{~mJ} / \mathrm{cm}^{2}$ and $\sigma_{x}{ }^{f}=1.24 \mathrm{~mm}$ ) is shown to depend both on the longitudinal $\left(\mathrm{d}_{2}\right)$ and transverse position of the LN crystal $\left(\mathrm{d}_{\mathrm{a}}\right)$. (d) Shows the asymmetric decrease in THz energy if the crystal position is offset from its transverse optimum which is found to be $d_{a} \approx 3-4 \mathrm{~mm}$ independent of the pump beam size.

\subsection{Finalization of the setup}

The last step of the optimization procedure allows adjustment of the vertical pump size $\sigma_{\mathrm{y}}{ }^{\mathrm{f}}$ via cylindrical optics before the setup $\left(\mathrm{M}_{\mathrm{y}}\right)$ such that the incident pulse energy illuminates the crystal with the fluence found to yield highest efficiency. With this final step, the total efficiency of the setup is finally maximized.

Fig 6(a) shows the spectrum of the THz pulses to be centered around $0.22 \mathrm{THz}$ for the room temperature setup. As the pump beam is elliptically shaped, it is not surprising that the recorded THz beam profile is slightly elliptical as well (see Fig. 6(b)). However, the measured THz beam sizes (1/e intensity radii) measured at different distances along z' are wellfitted by Gaussian beams with waists $\sigma_{\mathrm{x}}{ }^{\mathrm{THz}}$ and $\sigma_{\mathrm{y}}{ }^{\mathrm{THz}}$ and their waist set to the crystal output surface. Note, that the $\mathrm{THz}$ was propagating at an angle of $\sim 12^{\circ}$ with respect to the z' axis, which matches the expected angle calculated by Snell's law based on the difference between pulse-front tilt angle $\left(65.4^{\circ}\right)$ and crystal cut $\alpha\left(62.4^{\circ}\right)$. 
a)

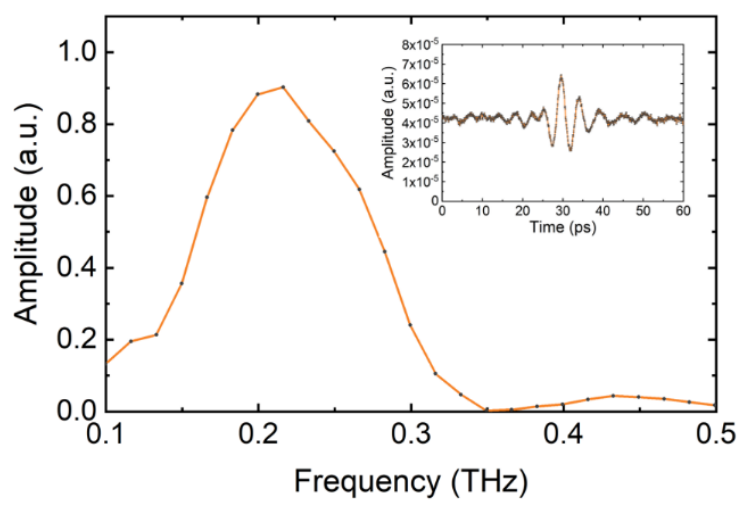

b)

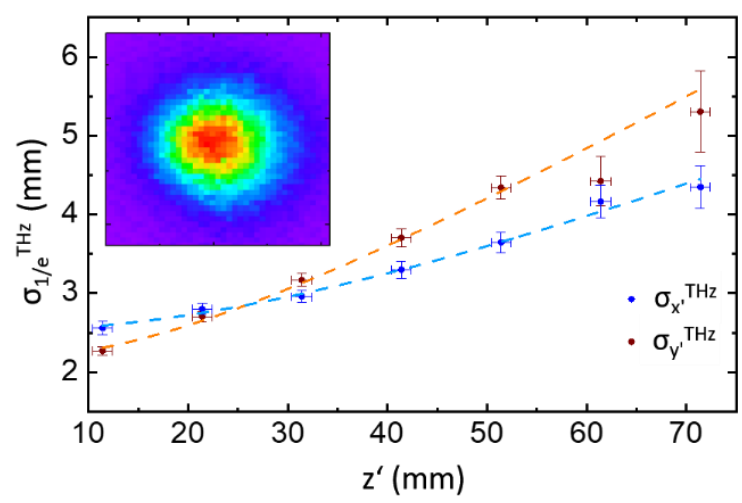

Figure 6. (a) Recorded spectrum of the finalized setup via a Michelson type interferometer. The interferogram is shown in the inset. (b) The measured $\mathrm{THz}$ beam sizes $\sigma_{\mathrm{x}}{ }^{\mathrm{THz}}$ and $\sigma_{\mathrm{y}}{ }^{\mathrm{THz}}$ (1/e intensity radii) along propagation $\mathrm{z}$ ' were fitted assuming Gaussian beam propagation (dashed lines). The THz beam profile recorded at closest distance to the crystal surface is depicted in the inset.

\subsection{Sensitivity of the conversion efficiency on the setup parameters}

The pulse front tilt angle $\gamma$ was observed to be a crucial parameter to be set correctly, as a small deviation of the grating angle $\theta_{\mathrm{i}}$ by only $1^{\circ}$ can lead to a drop of the efficiency by $\sim 60 \%$. As $\gamma$ is not observed to depend on the pump fluence significantly it thus can be adjusted at low pump fluence. This is advantageous in order to not optically damage the crystal accidentally during the procedure. As discussed, it is important to rather start with a larger beam size $\sigma_{\mathrm{x}}{ }^{\mathrm{f}}$ and the full beam incident on the crystal, in order to ensure $\gamma$ has the largest impact onto the efficiency. The pump polarization affects the efficiency just as severely but is experimentally more straightforward to optimize via a half-wave-plate. Furthermore, the beam size in the phase-matching plane sensitively affects the efficiency. While the efficiency first grows with increasing beam pump width, there is an optimum size after which the efficiency decreases slightly. Unfortunately, the beam size could not be expanded further to study this behavior in more detail as the size of our optics prevented even larger $1 / \mathrm{e}^{2}$ beam radii beyond $3 \mathrm{~mm}$. As expected, the crystal position along $\mathrm{x}$ and $\mathrm{z}$ should be changed simultaneously in order to optimize the interaction volume. However, if the whole pump beam is incident onto the crystal, the $\mathrm{x}$ and $\mathrm{z}$ positions impact the conversion efficiency less than the other parameters. In this case, a rough optimization can be performed by maximizing the THz energy via independent scans of $d_{2}$ and $d_{a}$. That optimization should be performed at high fluence, as the fluence level affects the effective interaction length of the nonlinear process.

\section{CONCLUSION AND OUTLOOK}

To conclude, a robust and systematic method to tune the tilted pulse-front setup, based on selected multi-dimensional parameter scans was introduced. The optimization strategy enables a straightforward determination of ideal parameters such as angular dispersion, temporal compression, beam size and pump fluence. From the data taken, the sensitivities on parameters were derived and discussed. Additionally, the characterization techniques discussed allow better comparability of different tilted pulse-front setups.

Such systematic optimization is important for the construction of high energy THz setups and can be applied for any nonlinear material used in such non-collinear phase-matching geometry. Thus, it is of special relevance for high field THz applications such as strong-field $\mathrm{THz}$ spectroscopy or charged particle acceleration. For example, the presented technique was used to construct and optimize two high energy setups in order to power a novel prototype of a THz electron gun, with first experiments yielding electron bunches with energies in the keV regime. Furthermore we note, that recently Bodrov and coworkers suggested improved performance of tilted-pulse front setups via a modified optimal imaging design in which image and crystal cut are at larger angle than the pulse front tilt, however requiring an outcoupling prism for the generated $\mathrm{THz}^{42}$. A systematic experimental study of their proposed design with comparison to our results may be of interest for future work. 


\section{ACKNOWLEDGEMENTS}

We gratefully thank D. Zhang for fruitful discussions on high-energy THz generation. For the initial laser design and implementation, we thank L. Zapata and M. Hemmer.

\section{REFERENCES}

[1] Koehl, R. M. and Nelson, K. A., "Terahertz polaritonics: automated spatiotemporal control over propagating lattice waves," Chemical Physics 267(1), 151 - 159 (2001).

[2] Feurer, T., Vaughan, J. C., and Nelson, K. A., "Spatiotemporal coherent control of lattice vibrational waves," Science 299(5605), 374-377 (2003).

[3] Fleischer, S., Zhou, Y., Field, R. W., and Nelson, K. A., "Molecular orientation and alignment by intense singlecycle THz pulses," Phys. Rev. Lett. 107, 163603 (2011).

[4] Kitano, K., Ishii, N., Kanda, N., Matsumoto, Y., Kanai, T., Kuwata-Gonokami, M., and Itatani, J., "Orientation of jet-cooled polar molecules with an intense single-cycle THz pulse," Phys. Rev. A 88, 061405 (2013).

[5] Sajadi, M., Wolf, M., and Kampfrath, T., "Terahertz-field-induced optical birefringence in common window and substrate materials," Opt. Express 23(22), 28985-28992 (2015).

[6] Lin, S., Yu, S., and Talbayev, D., "Measurement of quadratic terahertz optical nonlinearities using second-harmonic lock-in detection," Phys. Rev. Applied 10, 044007 (2018).

[7] Shen, Y., Watanabe, T., Arena, D. A., Kao, C.-C., Murphy, J. B., Tsang, T. Y., Wang, X. J., and Carr, G. L., "Nonlinear cross-phase modulation with intense single-cycle terahertz pulses," Phys. Rev. Lett. 99, 043901 (2007).

[8] Vicario, C., Shalaby, M., and Hauri, C. P., "Subcycle extreme nonlinearities in GaP induced by an ultrastrong terahertz field," Phys. Rev. Lett. 118, 083901 (2017).

[9] Pashkin, A., Sell, A., Kampfrath, T., and Huber, R., "Electric and magnetic terahertz nonlinearities resolved on the sub-cycle scale," New Journal of Physics 15(6), 065003 (2013).

[10]Lange, C., Maag, T., Hohenleutner, M., Baierl, S., Schubert, O., Edwards, E. R. J., Bougeard, D., Woltersdorf, G., and Huber, R., "Extremely nonperturbative nonlinearities in GaAs driven by atomically strong terahertz fields in gold metamaterials," Phys. Rev. Lett. 113, 227401 (2014).

[11]Zhang, D., Fallahi, A., Hemmer, M., Wu, X., Fakhari, M., Hua, Y., Cankaya, H., Calendron, A.-L., Zapata, L. E., Matlis, N. H., and Kärtner, F. X., "Segmented terahertz electron accelerator and manipulator (STEAM)," Nature Photonics 12(6), 336-342 (2018).

[12] Oh, T. I., Yoo, Y. J., You, Y. S., and Kim, K. Y., “Generation of strong terahertz fields exceeding $8 \mathrm{MV} / \mathrm{cm}$ at 1 kHz and real-time beam profiling," Applied Physics Letters 105(4), 041103 (2014).

[13] Oh, T. I., You, Y. S., Jhajj, N., Rosenthal, E. W., Milchberg, H. M., and Kim, K. Y., "Intense terahertz generation in two-color laser filamentation: energy scaling with terawatt laser systems," New Journal of Physics 15(7), 075002 (2013).

[14] Vaicaitis, V., Balachninaite, O., Morgner, U., and Babushkin, I., "Terahertz radiation generation by three-color laser pulses in air filament," Journal of Applied Physics 125(17), 173103 (2019).

[15] Cook, D. J. and Hochstrasser, R. M., "Intense terahertz pulses by four-wave rectification in air," Opt. Lett. 25(16), 1210-1212 (2000).

[16] Ropagnol, X., Kovács, Z., Gilicze, B., Zhuldybina, M., Blanchard, F., Garcia-Rosas, C. M., Szatmári, S., Földes, I. B., and Ozaki, T., "Intense sub-terahertz radiation from wide-bandgap semiconductor based large-aperture photoconductive antennas pumped by UV lasers," New Journal of Physics 21(11), 113042 (2019).

[17] You, D., Jones, R. R., Bucksbaum, P. H., and Dykaar, D. R., "Generation of high-power sub-single-cycle 500-fs electromagnetic pulses," Opt. Lett. 18(4), 290-292 (1993).

[18] Jones, R. R., You, D., and Bucksbaum, P. H., "Ionization of rydberg atoms by subpicosecond half-cycle electromagnetic pulses," Phys. Rev. Lett. 70, 1236-1239 (1993).

[19] Kampfrath, T., Battiato, M., Maldonado, P., Eilers, G., Nötzold, J., Mährlein, S., Zbarsky, V., Freimuth, F., Mokrousov, Y., Blügel, S., Wolf, M., Radu, I., Oppeneer, P. M., and Münzenberg, M., "Terahertz spin current pulses controlled by magnetic heterostructures," Nature Nanotechnology 8(4), 256-260 (2013).

[20] Seifert, T., Jaiswal, S., Sajadi, M., Jakob, G., Winnerl, S., Wolf, M., Kläui, M., and Kampfrath, T., "Ultrabroadband single-cycle terahertz pulses with peak fields of $300 \mathrm{kV} \mathrm{cm}-1$ from a metallic spintronic emitter," Applied Physics Letters 110(25), 252402 (2017). 
[21] Fülöp, J. A., Tzortzakis, S., and Kampfrath, T., "Laser-driven strong-field terahertz sources,” Advanced Optical Materials, 1900681 (2019).

[22]Hebling, J., Stepanov, A. G., Almási, G., Bartal, B., and Kuhl, J., “Tunable THz pulse generation by optical rectification of ultrashort laser pulses with tilted pulse fronts," Applied Physics B 78(5), 593-599 (2004).

[23] Auston, D. H., Cheung, K. P., Valdmanis, J. A., and Kleinman, D. A., "Cherenkov radiation from femtosecond optical pulses in electro-optic media," Phys. Rev. Lett. 53, 1555-1558 (1984).

[24] Kleinman, D. and Auston, D., "Theory of electrooptic shock radiation in nonlinear optical media," IEEE Journal of Quantum Electronics 20(8), 964-970 (1984).

[25] Koehl, R. M. and Nelson, K. A., "Coherent optical control over collective vibrations traveling at lightlike speeds," The Journal of Chemical Physics 114(4), 1443-1446 (2001).

[26] Hebling, J., Almási, G., Kozma, I. Z., and Kuhl, J., "Velocity matching by pulse front tilting for large-area THzpulse generation," Opt. Express 10(21), 1161-1166 (2002).

[27] Stepanov, A. G., Kuhl, J., Kozma, I. Z., Riedle, E., Almási, G., and Hebling, J., "Scaling up the energy of THz pulses created by optical rectification," Opt. Express 13(15), 5762-5768 (2005).

[28] Yeh, K.-L., Hoffmann, M. C., Hebling, J., and Nelson, K. A., "Generation of $10 \mu$ J ultrashort terahertz pulses by optical rectification," Applied Physics Letters 90(17), 171121 (2007).

[29] Stepanov, A. G., Bonacina, L., Chekalin, S. V., and Wolf, J.-P., "Generation of $30 \mu \mathrm{J}$ single-cycle terahertz pulses at $100 \mathrm{~Hz}$ repetition rate by optical rectification," Opt. Lett. 33(21), 2497-2499 (2008).

[30] Fülöp, J. A., Pálfalvi, L., Hoffmann, M. C., and Hebling, J., "Towards generation of mJ-level ultrashort THz pulses by optical rectification," Opt. Express 19(16), 15090-15097 (2011).

[31]Fülöp, J. A., Pálfalvi, L., Almási, G., and Hebling, J., "Design of high-energy terahertz sources based on optical rectification,” Opt. Express 18(12), 12311-12327 (2010).

[32] Hoffmann, M. C., Yeh, K.-L., Hebling, J., and Nelson, K. A., "Efficient terahertz generation by optical rectification at $1035 \mathrm{~nm}, "$ Opt. Express 15(18), 11706-11713 (2007).

[33]Fülöp, J. A., Pálfalvi, L., Klingebiel, S., Almási, G., Krausz, F., Karsch, S., and Hebling, J., “Generation of sub-mJ terahertz pulses by optical rectification," Opt. Lett. 37(4), 557-559 (2012).

[34] Bodrov, S. B., Murzanev, A. A., Sergeev, Y. A., Malkov, Y. A., and Stepanov, A. N., "Terahertz generation by tilted-front laser pulses in weakly and strongly nonlinear regimes," Applied Physics Letters 103(25), 251103 (2013).

[35] Vicario, C., Monoszlai, B., Lombosi, C., Mareczko, A., Courjaud, A., Fülöp, J. A., and Hauri, C. P., "Pump pulse width and temperature effects in lithium niobate for efficient THz generation," Opt. Lett. 38(24), 5373-5376 (2013).

[36] Shikata, J., Sato, M., Taniuchi, T., Ito, H., and Kawase, K., "Enhancement of terahertz-wave output from LiNbO3 optical parametric oscillators by cryogenic cooling," Opt. Lett. 24(4), 202-204 (1999).

[37]Huang, S.-W., Granados, E., Huang, W. R., Hong, K.-H., Zapata, L. E., and Kärtner, F. X., "High conversion efficiency, high energy terahertz pulses by optical rectification in cryogenically cooled lithium niobate," Opt. Lett. 38(5), 796-798 (2013).

[38] Fülöp, J. A., Ollmann, Z., Lombosi, C., Skrobol, C., Klingebiel, S., Pálfalvi, L., Krausz, F., Karsch, S., and Hebling, J., "Efficient generation of THz pulses with 0.4 mJ energy," Opt. Express 22(17), 20155-20163 (2014).

[39] Stepanov, A. G., Henin, S., Petit, Y., Bonacina, L., Kasparian, J., and Wolf, J.-P., "Mobile source of high-energy single-cycle terahertz pulses," Applied Physics B 101(1), 11-14 (2010).

[40] Wu, X., long Ma, J., Zhang, B., Chai, S., Fang, Z., Xia, C., Kong, D., Wang, J., Liu, H., Zhu, C., Wang, X., Ruan, C., and Li, Y., "Highly efficient generation of $0.2 \mathrm{~mJ}$ terahertz pulses in lithium niobate at room temperature with sub-50 fs chirped Ti:sapphire laser pulses," Opt. Express 26(6), 7107-7116 (2018).

[41] Tokodi, L., Hebling, J., and Pálfalvi, L., "Optimization of the tilted-pulse-front terahertz excitation setup containing telescope," Journal of Infrared, Millimeter, and Terahertz Waves 38(1), 22-32 (2017).

[42] Bodrov, S. B., Stepanov, A. N., and Bakunov, M. I., "Generalized analysis of terahertz generation by tilted-pulsefront excitation in a LiNbO3 prism," Opt. Express 27(3), 2396-2410 (2019).

[43] Pálfalvi, L., Fülöp, J. A., Almási, G., and Hebling, J., "Novel setups for extremely high-power single-cycle terahertz pulse generation by optical rectification," Applied Physics Letters 92(17), 171107 (2008).

[44] Kunitski, M., Richter, M., Thomson, M. D., Vredenborg, A., Wu, J., Jahnke, T., Schöffler, M., Schmidt-Böcking, H., Roskos, H. G., and Dörner, R., "Optimization of single-cycle terahertz generation in LiNbO3 for sub-50 femtosecond pump pulses,” Opt. Express 21(6), 6826-6836 (2013).

[45] Gustafson, T., Taran, J.-P., Kelley, P., and Chiao, R., "Self - modulation of picosecond pulses in electro - optic crystals," Optics Communications 2(1), 17 - 21 (1970). 
[46] Cronin-Golomb, M., "Cascaded nonlinear difference-frequency generation of enhanced terahertz wave production," Opt. Lett. 29(17), 2046-2048 (2004).

[47] Vodopyanov, K. L., "Optical generation of narrow-band terahertz packets in periodically-inverted electro-optic crystals: conversion efficiency and optimal laser pulse format," Opt. Express 14(6), 2263-2276 (2006).

[48] Jewariya, M., Nagai, M., and Tanaka, K., "Enhancement of terahertz wave generation by cascaded $\chi(2)$ processes in LiNbO3," J. Opt. Soc. Am. B 26(9), A101-A106 (2009).

[49] Nagai, M., Jewariya, M., Ichikawa, Y., Ohtake, H., Sugiura, T., Uehara, Y., and Tanaka, K., "Broadband and high power terahertz pulse generation beyond excitation bandwidth limitation via $\chi(2)$ cascaded processes in LiNbO3," Opt. Express 17(14), 11543-11549 (2009).

[50] Ravi, K., Huang, W. R., Carbajo, S., Wu, X., and Kärtner, F., "Limitations to THz generation by optical rectification using tilted pulse fronts," Opt. Express 22(17), 20239-20251 (2014).

[51] Bakunov, M. I., Bodrov, S. B., and Mashkovich, E. A., "Terahertz generation with tilted-front laser pulses: dynamic theory for low-absorbing crystals," J. Opt. Soc. Am. B 28(7), 1724-1734 (2011).

[52] Bartal, B., Kozma, I. Z., Stepanov, A. G., AlmÃ $;$ si, G., Kuhl, J., Riedle, E., and Hebling, J., "Toward generation of $\hat{\mathrm{I}}^{1 / 4 \mathrm{j}}$ range sub-ps thz pulses by optical rectification," Applied Physics B 86(3), 419-423 (2007).

[53] Ravi, K., Huang, W. R., Carbajo, S., Nanni, E. A., Schimpf, D. N., Ippen, E. P., and Kärtner, F. X., "Theory of terahertz generation by optical rectification using tilted-pulse-fronts," Opt. Express 23(4), 5253-5276 (2015).

[54]Zhong, S.-C., Zhai, Z.-H., Li, J., Zhu, L.-G., Li, J., Meng, K., Liu, Q., Du, L.-H., Zhao, J.-H., and Li, Z.-R., "Optimization of terahertz generation from LiNbO3 under intense laser excitation with the effect of three-photon absorption," Opt. Express 23(24), 31313-31323 (2015).

[55] Nagai, M., Matsubara, E., and Ashida, M., "High-efficiency terahertz pulse generation via optical rectification by suppressing stimulated Raman scattering process," Opt. Express 20(6), 6509-6514 (2012).

[56] Pálfalvi, L., Tóth, G., Tokodi, L., Márton, Z., Fülöp, J. A., Almási, G., and Hebling, J., "Numerical investigation of a scalable setup for efficient terahertz generation using a segmented tilted-pulse-front excitation," Opt. Express 25(24), 29560-29573 (2017).

[57] Tóth, G., Pálfalvi, L., Tibai, Z., Tokodi, L., Fülöp, J. A., Márton, Z., Almási, G., and Hebling, J., "Single-cycle scalable terahertz pulse source in reflection geometry," Opt. Express 27(21), 30681-30691 (2019).

[58] Ofori-Okai, B. K., Sivarajah, P., Huang, W. R., and Nelson, K. A., “THz generation using a reflective stair-step echelon," Opt. Express 24(5), 5057-5068 (2016).

[59] Hirori, H., Doi, A., Blanchard, F., and Tanaka, K., "Single-cycle terahertz pulses with amplitudes exceeding 1 MV/cm generated by optical rectification in LiNbO3," Applied Physics Letters 98(9), 091106 (2011).

[60] Wu, X., Calendron, A.-L., Ravi, K., Zhou, C., Hemmer, M., Reichert, F., Zhang, D., Cankaya, H., Zapata, L. E., Matlis, N. H., and Kärtner, F. X., "Optical generation of single-cycle $10 \mathrm{MW}$ peak power $100 \mathrm{GHz}$ waves," Opt. Express 24(18), 21059-21069 (2016).

[61] Gayer, O., Sacks, Z., Galun, E., and Arie, A., “Temperature and wavelength dependent refractive index equations for MgO-doped congruent and stoichiometric LiNbO3," Applied Physics B 91(2), 343-348 (2008).

[62] Jundt, D. H., "Temperature-dependent Sellmeier equation for the index of refraction, n_e, in congruent lithium niobate," Opt. Lett. 22, 1553-1555 (Oct 1997).

[63] Cankaya, H., Demirbas, U., Hua, Y., Hemmer, M., Zapata, L. E., Pergament, M., and Kaertner, F. X., “190-mJ cryogenically-cooled Yb:YLF amplifier system at $1019.7 \mathrm{~nm}$," OSA Continuum 2(12), 3547-3553 (2019).

[64] Demirbas, U., Cankaya, H., Hua, Y., Thesinga, J., Pergament, M., and Kärtner, F. X., "20-mJ, sub-ps pulses at up to $70 \mathrm{~W}$ average power from a cryogenic Yb:YLF regenerative amplifier," Opt. Express 28, 2466-2479 (Jan 2020).

[65] Bakunov, M. I., Bodrov, S. B., and Tsarev, M. V., "Terahertz emission from a laser pulse with tilted front: Phasematching versus Cherenkov effect," Journal of Applied Physics 104(7), 073105 (2008).

[66] Wu, X., Zhou, C., Huang, W. R., Ahr, F., and Kärtner, F. X., "Temperature dependent refractive index and absorption coefficient of congruent lithium niobate crystals in the terahertz range," Opt. Express 23, 29729-29737 (Nov 2015).

[67] Pálfalvi, L., Hebling, J., Kuhl, J., Péter, A., and Polgár, K., “Temperature dependence of the absorption and refraction of $\mathrm{Mg}$-doped congruent and stoichiometric LiNbO3 in the THz range," Journal of Applied Physics 97(12), 123505 (2005).

[68] Wang, L., Kroh, T., Matlis, N. H., and Kaertner, F., "Full 3D+1 modelling of tilted-pulse-front setups for singlecycle terahertz generation." arXiv (2019).

[69] Hebling, J., Yeh, K.-L., Hoffmann, M. C., Bartal, B., and Nelson, K. A., “Generation of high-power terahertz pulses by tilted-pulse-front excitation and their application possibilities," J. Opt. Soc. Am. B 25(7), B6-B19 (2008). 